\title{
ESTUDO COMPARATIVO ENTRE AS TÉCNICAS DE NISSEN E BOIX-OCHOA EM RATOS
}

\author{
COMPARATIVE STUDY BETWEEN THE TECHNIQUES OF THE NISSEN AND BOIX- \\ OCHOA IN RATS
}

\author{
Karla F. Pinto1; Saulo Marcos R. Ferrante²; Ivonete Siviero²; \\ Ivens B. Meio ${ }^{3}$; Marcos Antônio Turcatel ${ }^{1}$; Danielle Forny ${ }^{1}$
}

\begin{abstract}
RESUMO: Objetivo: Comparar, em ratos, a eficiência das técnicas de Nissen e Boix-Ochoa no tratamento do refluxo gastroesofagiano (RGE) induzido pela operação de Heller. Método: Foram usados 30 ratos Wistar, com idades entre 40 e 60 dias de vida e peso corporal entre $210 \mathrm{~g} \mathrm{e} 342 \mathrm{~g}$. Os animais foram distribuídos em três Grupos (A, B, C) de 10 ratos. Em todos os animais realizou-se a operação de Heller. No Grupo B ela foi seguida de uma operação de Nissen e, no Grupo C, de uma operação de Boix-Ochoa. A pressão intra-gástrica necessária para produzir RGE foi medida em todos os animais: antes de qualquer procedimento; imediatamente após as operações de Heller, Nissen e Boix-Ochoa; e seis semanas depois, quando os animais foram mortos. Resultados: Verificou-se que tanto a recuperação da perda de peso, que caracteriza o quadro de RGE, como a reconstituição mais fisiológica dos gradientes pressóricos gastroesofagianos são obtidos de forma mais eficiente pela técnica de Boix- Ochoa. Conclusão: As operações de Nissen e Boix- Ochoa são eficientes no tratamento do RGE induzido pela técnica de Heller, em ratos. A segunda, no entanto, restaurou, de forma mais adequada, os valores fisiológicos dos parâmetros estudados neste trabalho: o peso corporal e os gradientes pressóricos gastroesofagianos (Rev. Col. Bras. Cir. 2007; 34(6): 407-411).
\end{abstract}

Descritores: Refluxo gastroesofágico; Fundoplicatura/métodos; Procedimentos cirúrgicos operatórios; Ratos Wistar.

\section{INTRODUÇÃO}

Define-se refluxo gastroesofagiano (RGE) como a enfermidade causada pela movimentação do conteúdo gástrico para o esôfago, decorrente de um defeito anátomo-funcional da junção esôfago-gástrica (JEG). Este termo é pouco preciso, uma vez que o refluxo do conteúdo gástrico para o esôfago é um fenômeno normal em grande número de espécies e, em humanos, na quase totalidade dos indivíduos, especialmente crianças.

A partir da década de 50, começou-se a compreender melhor os mecanismos fisiopatológicos envolvidos na produção de RGE, especialmente com a definição da estrutura anatômica, denominada de esfíncter esofagiano inferior (EEI), considerado o mecanismo central da chamada barreira antirefluxo gastroesofágica (BARGE) $)^{1-6}$. Isto permitiu o desenvolvimento de técnicas propedêuticas que mostraram que o RGE é um fenômeno bem mais freqüente que se pensava até então ${ }^{7-10}$

O tratamento inicial do RGE é, consensualmente, clínico e se baseia em medidas anti-refluxo, como orientação postural e dietética, além do uso de drogas do tipo procinético, colinérgico e anti-ácido. Quando o problema é, clinicamente, insolúvel, uma opção cirúrgica deve ser considerada ${ }^{11-15}$.
Apesar da proposta de muitas técnicas cirúrgicas, ao longo das últimas décadas, para tratar o RGE, atualmente duas, apenas, congregam as preferências dos mais importantes centros médicos pediátricos mundiais: são as técnicas de Nissen e de Boix-Ochoa ${ }^{16,17}$. Ambas se baseiam em critérios anátomo-funcionais de reconstrução da BARGE, mas os resultados finais de ambas têm sido criticados.

Para comparar e, se possível, eleger a técnica cirúrgica que trata com mais eficiência o RGE em animais de pequeno porte, idealizou-se um trabalho experimental em ratos em que se produz, uniformemente, a quebra da BARGE pela confecção de uma cardiomiotomia a Heller e, distribuindo-se os animais em dois Grupos, utilizou-se, em um deles, a técnica de Nissen e, no outro, a de Boix-Ochoa.

\section{MÉTODO}

Foram utilizados 30 ratos Wistar, machos e fêmeas adultos, com idades entre 40 e 60 dias de vida e peso corporal entre $210 \mathrm{~g} \mathrm{e} 342 \mathrm{~g}$. Os animais foram mantidos no biotério do Laboratório de Cirurgia Experimental (LCE) da Faculdade de Ciências Médicas da Universidade do Estado do Rio de Janeiro - UERJ e, sob supervisão veterinária, receberam água e ração próprias para roedores durante o estudo.

\footnotetext{
1. Mestre em Cirurgia pela UFRJ.

2. Professor Adjunto do Departamento de Cirurgia da UFRJ.

3. Doutor em Cirurgia pela UFRJ.

Recebido em 30/03/2007

Aceito para publicação em 30/05/2007

Conflito de interesses: nenhum

Fonte de financiamento: nenhuma

Trabalho realizado no Laboratório de Cirurgia Experimental (LCE) da Faculdade de Ciências Médicas da Universidade do Estado do Rio de Janeiro UERJ.
} 
Foram distribuidos, de forma randomizada, em três Grupos de 10 ratos. No primeiro (A), ou Grupo de controle, realizou-se a operação de Heller. No segundo (B), as operações de Heller e de Nissen. E, no terceiro (C), as de Heller e Boix-Ochoa. Todos eles foram anestesiados com éter sulfúrico por via inalatória, presos na prancha cirúrgica em posição de decúbito dorsal e submetidos à depilação abdominal. $\mathrm{O}$ acesso à cavidade peritoneal era feito por meio de uma incisão longitudinal mediana de, aproximadamente, $5 \mathrm{~cm}$. No inventário da cavidade, via-se o estômago e fazia-se a obstrução do piloro por meio de um dreno de Penrose $n^{\circ} 1$. Realizavam-se, então, duas punções no antro gástrico com dois cateteres Jelco 22, acoplados, cada um, a uma borracha de equipo de soro com $30 \mathrm{~cm}$ de comprimento. Numa delas, era conectado um manômetro de pressão e, na outra, uma pêra manual de insuflação aérea.

Fazia-se, então, a insuflação do estômago pelo sistema conectado à pêra e observava-se o valor mínimo de pressão intra-gástrica capaz de produzir refluxo aéreo gastroesofagiano. Este valor, presumidamente registrado no momento imediatamente anterior à abertura da JEG, era definido pela máxima pressão alcançada antes da queda abrupta da pressão intra-gástrica registrada pelo marcador manométrico, e considerado o limite fisiológico de eficiência da BARGE, ou pressão fisiológica (PF).

Os animais eram submetidos, então, a uma cardiomiotomia a Heller, que consiste na abertura longitudinal da musculatura do esôfago terminal, preservando-se a mucosa do órgão. Em seguida, era medida, novamente, a pressão intragástrica mínima capaz de permitir o RGE, ou pressão pós-Heller $(\mathrm{PPH})$.

A partir deste momento, formavam-se os três grupos. No grupo A, de controle, o procedimento era encerrado neste ponto e fechava-se a cavidade abdominal com fio Monocryl 4.0. No Grupo B, realizava-se a cirurgia de Nissen, com o esôfago calibrado por uma sonda esofágica $n^{\circ} 8$. O fundo gástrico era passado por trás do esôfago terminal e suturado ao próprio estômago, anteriormente. A fundoplicatura era feita com dois pontos separados de fio inabsorvível (Prolene 4.0) que passavam, ambos, pelo estômago, camada muscular do esôfago em sua face anterior e, novamente, pelo estômago. O esôfago terminal ficava, assim, envolto pelo fundo gástrico, criando o mecanismo valvular anti-refluxo descrito por Nissen ${ }^{16}$. A cavidade abdominal era, então, fechada com fio Monocryl 4.0. No Grupo C, realizava-se a técnica de BoixOchoa, também com o esôfago calibrado por uma sonda esofágica $n^{\circ} 8$. Nesta operação, a fundoplicatura era feita pela fixação, com Prolene 4.0, do fundo gástrico à parede lateral direita do esôfago com três pontos separados, e ao diafragma com um único ponto, à esquerda do hiato esofagiano. Nos dois Grupos, B e C, antes da realização da fundoplicatura, era feita uma aproximação dos pilares paraesofagianos com um ponto simples de Prolene 4.0. Em todos os animais, a parede abdominal foi fechada com Monocryl 4.0, e os ratos eram colocados em suas gaiolas, onde recebiam água e ração, $a d$ libitum, por seis semanas.

Todos os animais foram pesados semanalmente. Após este tempo, foram anestesiados e novamente submetidos à laparotomia para medir, como descrito acima, a pressão intragástrica mínima capaz de produzir refluxo aéreo gastroesofágico, ou pressão final (PFinal). Todos foram mortos, a seguir, por meio de superdosagem de éter inalatório até a parada cárdio-respiratória.

Foram utilizados dois parâmetros de avaliação da eficiência do procedimento realizado: a variação do peso dos ratos e da pressão intra-gástrica. Calculou-se a média aritmética, mediana, desvio padrão, valores máximo e mínimo de cada grupo e, em relação à pressão intra-gástrica, os primeiro e terceiro quartis e a média de tendência central. Os resultados foram submetidos ao teste $\mathbf{t}$ para obtenção do $\mathbf{p}$-valor, tendo sido considerados significativos os valores de $\mathbf{p}$ menores que 0,05 .

Este trabalho foi realizado de acordo com a Lei $\mathrm{n}^{\circ}$ 6.638, de 08 de maio de 1979, referente aos princípios éticos do uso de animais em experiência científica.

\section{RESULTADOS}

Um dos ratos do Grupo A morreu no segundo dia de pós-operatório por causa de broncoaspiração, e outro durante a terceira semana, devido a uma virose comum em ratos de laboratório, a neuropatia linfocítica. No Grupo B, um rato morreu no terceiro dia de pós-operatório por motivo desconhecido, tendo sido a necropsia inconclusiva. No Grupo C não houve óbito nesta fase.

Comparados os valores de peso corporal dos animais antes de qualquer procedimento com o peso após seis semanas da operação (peso final), observou-se o seguinte (tabela 1): no Grupo A, três ratos perderam e cinco ganharam peso, verificando-se média de ganho ponderal de $4,88 \mathrm{~g}$. No Grupo B, um rato perdeu e oito ganharam peso, com média de $28,44 \mathrm{~g}$ de ganho ponderal. Finalmente, no Grupo C, todos os ratos ganharam peso, com média de $62,60 \mathrm{~g}$.

Com relação aos valores pressóricos intra-gástricos necessários para produzir o RGE (tabela 2), verificou-se que, no Grupo A, a operação de Heller foi eficiente, provocando o

Tabela 1 - Ganho ponderal (g).

\begin{tabular}{lrcl}
\hline & Média & Variação & p-valor \\
\hline Grupo A & 4,88 & $-10 /+22$ & 0,283 \\
Grupo B & 28,44 & $-14 /+90$ & 0,019 \\
Grupo C & 62,60 & $+30 /+100$ & 0,0001 \\
Grupos B/C & & & 0,017 \\
\hline
\end{tabular}

Tabela 2 - Média da pressão intra-gástrica ( $\mathrm{mm} \mathrm{Hg}$ ).

\begin{tabular}{lccc}
\hline & PF & PFinal & p-valor \\
\hline Grupo A & 71 & 28,75 & 0,0000057 \\
Grupo B & 63 & 162,22 & 0,0000201 \\
Grupo C & 73 & 65 & 0,4279744 \\
Grupos B/C & - & - & 0,0000003 \\
(PFinal) & & & \\
\hline
\end{tabular}


refluxo e mantendo, após seis semanas de pós-operatório, um valor pressórico inferior àquele considerado fisiológico. Em média, esses valores foram de $71 \mathrm{~mm} \mathrm{Hg}$ para a PF e de 28,75 $\mathrm{mm} \mathrm{Hg}$ para a PFinal. No Grupo B, em que a média da PF foi de $63 \mathrm{mmHg}$, verificou-se que a média da PFinal elevou-se para 162,22 mm Hg. E, no Grupo C, a média da PF foi de $73 \mathrm{~mm} \mathrm{Hg} \mathrm{e}$ a da PFinal $65 \mathrm{~mm} \mathrm{Hg}$.

\section{DISCUSSÃO}

Nas últimas décadas, várias técnicas cirúrgicas foram desenvolvidas para tratar o RGE. Atualmente, a fundoplicatura de Nissen é o procedimento mais utilizado, sendo consensual sua alta eficiência no controle do RGE, com a eliminação dos sinais e sintomas da doença e o desenvolvimento normal da criança ${ }^{18-21}$. Contudo, muitos trabalhos de revisão associaram complicações importantes à fundoplicatura de Nissen, especialmente disfagia, incapacidade de vomitar e distensão gástrica permanente, todas relacionadas à grande competência do mecanismo valvular construído cirurgicamente. Além disso, esta verdadeira vedação do estômago foi, também, associada, nos casos mais graves, à própria perfuração do órgão, à formação de hérnias paraesofagianas, à migração da válvula para o tórax e à invaginaçãp esôfago-gástrica ${ }^{22-28}$.

Na tentativa de evitar as complicações relacionadas à fundoplicatura de Nissen, alguns autores desenvolveram outras técnicas cirúrgicas, dando prioridade à construção de mecanismos valvulares menos eficazes e mais próximos da normalidade anátomo-funcional da JEG.

Mesmo admitindo os bons resultados anti-refluxo obtidos com a técnica de Nissen, Boix-Ochoa et al 2,17,29,30 consideraram-na inadequada para crianças e propuseram, em 1983, uma nova técnica para o tratamento do RGE, baseada no restabelecimento de parâmetros anátomo-funcionais, enfatizando a necessidade da restauração do ângulo de His e do alongamento do esôfago intra-abdominal. Comparados com a fundoplicatura de Nissen, seus resultados mostraram um número menor de complicações, uma vez que não havia distensão da câmara gástrica e a criança não perdia a capacidade de vomitar e eructar, embora não produzisse RGE em condições normais. Também não foram relatadas, com esta técnica, a herniação do esôfago intraabdominal, juntamente com o estômago, para o tórax, nem perfuração gástrica por obstrução em alça fechada ${ }^{18}$.

Muitos centros médicos começaram, a partir desses resultados, a preferir a técnica de Boix-Ochoa para o tratamento do RGE, embora continuasse a ser consensual a utilização da técnica de Nissen em crianças com alterações neurológicas graves, em virtude da falta de defesa desses pacientes contra a broncoaspiração de conteúdo gástrico. Nestes casos, é necessário que a operação anti-refluxo funcione como um mecanismo que o impossibilite ${ }^{21}$. A falta de embasamento teórico para definir a preferência por uma ou outra técnica motivou a elaboração deste estudo experimental.

Em todos os Grupos, observou-se ganho ponderal em seis semanas. No Grupo A, no entanto, a média de 4,88 g não teve significância estatística (p > 0,05), demonstrando que a operação de Heller mantém, pelo menos por este prazo, RGE, o que reduz, como se observa clinicamente, a possibilidade de ganho ponderal expressivo. No Grupo B, a média de $28,44 \mathrm{~g}$, com $\mathbf{p}<0,05$, mostrou a eficiência da operação de Nissen no tratamento do RGE. No Grupo C, este resultado foi ainda mais expressivo, com a média de ganho ponderal chegando a $62,60 \mathrm{~g}$ e o valor de $\mathbf{p}$ sendo igual a 0,0001 . A comparação entre os Grupos B e C mostrou diferença estatística significativa em favor da técnica de Boix-Ochoa, com $\mathbf{p}<0,05$.

Verifica-se, portanto, que por este parâmetro, os ratos submetidos à operação de Boix-Ochoa têm um desenvolvimento melhor, uma vez que a quantidade e a qualidade dos alimentos oferecidos foram iguais. Por outro lado, sabe-se que o RGE em crianças se caracteriza pela perda de peso dos pacientes, assim como se tem observado, clinicamente, ganho ponderal com a correção do refluxo, seja pela técnica de Nissen, seja pela de Boix-Ochoa. Os resultados deste estudo confirmam, no rato, esta observação feita em humanos e permitem formular a hipótese de que a operação de Boix-Ochoa, melhor que a de Nissen, atinge este objetivo.

A avaliação da pressão intra-gástrica, antes e depois da realização de qualquer procedimento cirúrgico que comprometesse a eficiência da JEG, permitiu a verificação dos valores pressóricos fisiológicos, pós Heller, pós Nissen e pós Boix-Ochoa. A diferença entre as pressões fisiológica e pós Heller (Grupo A) demonstra que esta técnica cirúrgica é eficiente para produzir RGE no rato, podendo ser utilizada como um procedimento capaz de quebrar a BARGE.

No Grupo B, verificou-se que, nos nove ratos submetidos à operação de Nissen e que completaram seis semanas de pós operatório, as pressões finais ficaram muito acima da média da pressão fisiológica. A variância no Grupo foi muito alta $(894,44 \mathrm{~mm} \mathrm{Hg})$, produzindo um desvio padrão de 23,91 $\mathrm{mm} \mathrm{Hg}$, o que significa, do ponto de vista estatístico, que nenhum animal deste Grupo apresentou pressão intra-gástrica considerada fisiológica. Já nos ratos submetidos à técnica de Boix-Ochoa, (Grupo C), oito ficaram abaixo e dois acima da média da pressão fisiológica.

Neste mesmo Grupo C, a média da pressão fisiológica foi de $73 \mathrm{~mm} \mathrm{HG}$ e a da pressão final $65 \mathrm{~mm} \mathrm{Hg}$. A variância foi de 494,44 mm Hg em relação à pressão final, com um desvio padrão de 22,24 mm Hg, mostrando que sete ratos apresentavam uma pressão final considerada fisiológica, um rato apresentava-a abaixo da normalidade e dois ratos acima.

Avaliando-se o p valor, verifica-se que, nos três Grupos, a operação de Heller foi eficaz na produção de RGE. Mas, analisando-se os valores referentes à comparação entre as pressões fisiológica e final nos Grupos A e B, observa-se que há uma diferença com significância estatística. Já no Grupo C, o $\mathbf{p}$ valor mostra que a diferença entre as pressões fisiológica e final não tem significado estatístico. Este dado sugere que tal diferença pode vir a fundamentar, se confirmada em crianças, uma conduta clínica, como a escolha da técnica de BoixOchoa no tratamento do RGE.

As operações de Nissen e Boix- Ochoa são eficientes no tratamento do RGE induzido pela técnica de Heller, em 
ratos. A segunda, no entanto, restaurou, de forma mais adequada, os valores fisiológicos dos parâmetros estudados neste trabalho: o peso corporal e os gradientes pressóricos gastroesofagianos.

\begin{abstract}
Objective: To compare, in rats, the efficiency of Nissen and Boix-Ochoa techniques in the treatment of gastroesophageal reflux (GER) induced by a Heller's procedure. Method: 30 rats Wistar aged between 40 and 60 days and weighing between $210 \mathrm{~g}$ and $342 \mathrm{~g}$ were used. The animals were divided in three $(A, B, C)$ groups of 10 rats each. A Heller's operation was made in all the animals. In group B, it was followed by a Nissen procedure and, in group C, by a BoixOchoa procedure. The intragastric pressure necessary to produce reflux was mesured in all animals: before any procedure; immediately after the Heller, Nissen and Boix-Ochoa porcedures; and 6 weeks later, when the animals were killed. Results: Restauration of body weight and reconstitution of more physiologic gastroesophageal pressure gradients were more effective with the Boix-Ochoa technique. Conclusion: the techniques of Nissen and Boix-Ochoa are effective in the treatment of GER induced by a Heller's procedure in rats. The second one, howerver, restaures, more adequately, the physiologic values of the two parameters used in this study: body weight and gastroesophageal pressure gradients.
\end{abstract}

Key words: Gastroesophageal reflux; Fundoplication/methods; Surgical procedures, operative; Rats, Wistar.

\section{REFERÊNCIAS}

1. Allison PR. Reflux esophagitis, sliding hiatal hernia, and the anatomy of repair. Surg Gynecol Obstet. 1951;92(4):419-31.

2. Boix-Ochoa J, Marhuenda C. Gastroesophageal reflux. In: Ashcraft KW, Holder TM. Pediatric surgery. $5^{\text {th }}$ ed. Philadelphia: WB Saunders; 1999. p. 370-85.

3. Capella MR. Refluxo gastroesofágico. In: Macksoud JG. Cirurgia pediátrica. $1^{a}$ ed. Rio de Janeiro: Revinter; 1998. p. 530-40.

4. Creamer B, Pierce JW. Observations on the gastroesophageal junction during swallowing and drinking. Lancet. 1957;273(7009):1309-12.

5. Hillemeier AC. Gastroesophageal reflux. Diagnostic and therapeutic approaches. Pediatr Clin North Am. 1996;43(1):197-12.

6. Winans CS, Harris LD. Quantitation of lower esophageal sphincter competence. Gastroenterology. 1967;52(5):773-8.

7. Boix-Ochoa J, Rowe MI. Gastroesophageal reflux. In: Welch KJ, Randolph JG, Ravitch MM, O’Neill JA Jr, Rowe MI, editors. Pediatric surgery. $5^{\text {th }}$ ed. St. Louis: Mosby; 1998. p. 1007-28.

8. Demeester TR, Wernly JA, Bryant GH, Little AG, Skinner DB. Clinical and in vitro analysis of determinants of gastroesophageal competence. A study of the principals of antireflux surgery. Am J Surg. 1979;137(1):39-46.

9. Doods WJ, Hogan WJ, Helm JF, Dent J. Pathogenesis of reflux esophagitis. Gastroenterology. 1981;81(2):376-94.

10. Jolley SG. Current surgical considerations in gastroesophageal reflux disease in infancy and childhood. Surg Clin North Am. 1992;72(6):1365-91.

11. Pettersson GB, Bombeck CT, Nyhus LM. The lower esophageal sphincter: mechanisms of opening and closure. Surgery. 1980;88(2):307-14

12. Johnson DG, Jolley SG. Gastroesophageal reflux in infants and children. Recognition and treatment. Surg Clin North Am. 1981;61(5):1101-15.

13. Pearl RH, Robie DK, Ein SH, Shandling B, Wesson DE, Superina R, Mctaggart K, Garcia VF, O'Connor JA, Filler RM. Complications of gastroesophageal antireflux surgery in neurologically impaired versus neurologically normal children. J Pediatr Surg. 1990;25(11):1169-73.
14. Pennel RC, Lewis JE, Cradock TV, Danis RK, Kaminski DL. Management of severe gastroesophageal reflux in children. Arch Surg. 1984;119(5):553-7.

15. Peter CS, Sprodowski N, Bohnhorst B, Silny J, Poets CF. Gastroesophageal reflux and apnea of prematurity: no temporal relationship. Pediatrics. 2002;109(1):8-11.

16. Nissen R. Gastropexy and "fundoplication" in surgical treatment of hiatal hernia. Am J Dig Dis. 1961;6(10):954-61.

17. Boix-Ochoa J. The physiologic approach to the management of gastric esophageal reflux. J Pediatr Surg. 1986;21(12):1032-9.

18. Cohen Z, Fishman S, Yulevich A, Kurtzbart E, Mares AJ. Nissen fundoplication and Boix-Ochoa antireflux procedure: comparison between two surgical techniques in the treatment of gastroesophageal reflux in children. Eur J Pediatr Surg. 1999;9(5):289-93.

19. Kawahara H, Imura K, Yagi M, Yoneda A, Soh H, Tazuke Y, Okada A. Mechanisms underlying the antireflux effect of Nissen fundoplication in children. J Pediatr Surg. 1998;33(11):1618-22.

20. Randolph J. Experience with the Nissen fundoplication for correction of gastroesophageal reflux in infants. Ann Surg. 1983;198(5):579-84.

21. Smith CD, Othersen HB Jr, Gogan NJ, Walker JD. Nissen fundoplication in children with profound neurologic disability. High risks and unmet goals. Ann Surg. 1992;215(6):654-8; discussion 658-9.

22. Alrabeeah A, Giacomantonio M, Gillis DA. Paraesophageal hernia after Nissen fundoplication: a real complication in pediatric patients. J Pediatr Surg. 1988;23(8):766-8.

23. Dedinsky GK, Vane DW, Black T, Turner MK, West KW, Grosfeld JL. Complications and reoperation after Nissen fundoplication in childhood. Am J Surg. 1987;153(2):177-83.

24. Festen C. Paraesophageal hernia: a major complication of Nissen's fundoplication. J Pediatr Surg. 1981;16(4):496-9.

25. Jolley SG, Tunell WP, Hoelzer DJ, Smith EI. Postoperative small bowel obstruction in infants and children: a problem following Nissen fundoplication. J Pediatr Surg. 1986;21(5):407-9.

26. Kazerooni NL, VanCamp J, Hirschl RB, Drongowski RA, Coran AG. Fundoplication in 160 children under 2 years of age. J Pediatr Surg. 1994;29(5):677-81. 
27. Post PJM, Robben SGF, Meradji M. Gastro-oesophageal intussusception after Nissen-fundoplication. Pediatr Radiol. 1990;20(4):282.

28. Turnage RH, Oldham KT, Coran AG, Blane CE. Late results of fundoplication for gastroesophageal reflux in infants and children. Surgery. 1989;105(4):457-64.

29. Bardaji C, Boix-Ochoa J. Contribution of the His angle to the gastroespphageal antireflux mechanism. Pediatr Surg Int. 1986;1(3):172-6.

30. Boix-Ochoa J, Canals J. Maturation of the lower esophagus. J Pediatr Surg. 1976;11(5):749-56.
Como citar este artigo:

Pinto KF, Ferrante SMR, Siviero I, Méio IB, Turcatel MA, Forny

D. Estudo comparativo da competência anti-refluxo entre as técnicas de Nissen e Boix-Ochoa em ratos. Rev Col Bras Cir. [periódico na Internet] 2007; 34(6). Disponível em URL: http://www.scielo.br/ rcbc

Endereço para correspondência:

Ivonete Siviero

E-mail: ivonetesiviero@terra.com.br 